\title{
Integral Operator Defined by k-th Hadamard Product
}

\author{
Maslina Darus \& Rabha W. Ibrahim \\ School of Mathematical Sciences \\ Faculty of science and Technology, Universiti Kebangsaan Malaysia \\ Bangi 43600, Selangor Darul Ehsan, Malaysia \\ Email: maslina@ukm.my, rabhaibrahim@yahoo.com
}

\begin{abstract}
We introduce an integral operator on the class $A$ of analytic functions in the unit disk involving $k$-th Hadamard product (convolution) corresponding to the differential operator defined recently by Al-Shaqsi and Darus. New classes containing this operator are studied. Characterization and other properties of these classes are studied. Moreover, subordination and superordination results involving this operator are obtained.
\end{abstract}

Keywords: Hadamard product; integral operator; subordination; superordination.

AMS Mathematics Subject Classification (2000): 30C45.

\section{$1 \quad$ Introduction}

Let $\mathrm{H}$ be the class of functions analytic in the unit disk $U$ and $\mathrm{H}[a, n]$ be the subclass of $\mathrm{H}$ consisting of functions of the form $f(z)=a+a_{n} z^{n}+a_{n+1} z^{n+1}+\ldots$ Let $\mathrm{A}$ be the subclass of $\mathrm{H}$ consisting of functions of the form

$$
f(z)=z+\sum_{n=2}^{\infty} a_{n} z^{n}, z \in U .
$$

The following differential operator is defined in [1] and studied in [2] $\mathrm{D}_{\lambda, \delta}^{k}: \mathrm{A} \rightarrow \mathrm{A}$ by

$$
\mathrm{D}_{\lambda, \delta}^{k} f(z)=z+\sum_{n=2}^{\infty}[1+(n-1) \lambda]^{k} C(\delta, n) a_{n} z^{n}, k \in \mathrm{N} \cup\{0\}, \lambda \geq 0, \delta \geq 0,
$$

where

Received April 21 $1^{\text {st }}, 2010$, Revised May 24 ${ }^{\text {th }}, 2010$, Accepted for publication May $25^{\text {th }}, 2010$. 


$$
C(\delta, n)=\left(\begin{array}{c}
n+\delta-1 \\
\delta
\end{array}\right)=\frac{\Gamma(n+\delta)}{\Gamma(n) \Gamma(\delta+1)} .
$$

Remark 1.1. When $\lambda=1, \delta=0$ we get $\mathrm{S}$ ă lă gean differential operator [3], $k=0$ gives Ruscheweyh operator [4], $\delta=0$ implies Al-Oboudi differential operator of order (k) [5] and when $\lambda=1$ operator (2) reduces to Al-shaqsi and Darus differential operator of order (k) [6].

Given two functions $f, g \in \mathrm{A}, f(z)=z+\sum_{n=2}^{\infty} a_{n} z^{n} \quad$ and $g(z)=z+\sum_{n=2}^{\infty} b_{n} z^{n}$ their convolution or Hadamard product $f(z) * g(z)$ is defined by

$$
f(z) * g(z)=z+\sum_{n=2}^{\infty} a_{n} b_{n} z^{n}, z \in U
$$

And for several functions $f_{1}(z), \ldots, f_{m}(z) \in \mathrm{A}$

$$
f_{1}(z) * \ldots * f_{m}(z)=z+\sum_{n=2}^{\infty}\left(a_{1 n} \ldots a_{m n}\right) z^{n},, z \in U
$$

Analogous to $\mathrm{D}_{\lambda, \delta}^{k} f(z), z \in U$ we define an integral operator $\mathrm{J}_{\lambda, \delta}^{k}: \mathrm{A} \rightarrow \mathrm{A}$ as follows.

Let

$$
\begin{aligned}
& \begin{aligned}
& \phi(z):= \frac{z}{1-z}+\frac{\lambda z}{(1-z)^{2}}-\frac{\lambda z}{1-z}, \lambda \geq 0 . \\
& F_{k}(z)=\underbrace{\phi(z) * \ldots * \phi(z)}_{k \text {-times }} *\left[\frac{z}{(1-z)^{\delta+1}}\right] \\
& \quad=z+\sum_{n=2}^{\infty}[1+(n-1) \lambda]^{k} C(\delta, n) z^{n}, k \in \mathrm{N}_{0} .
\end{aligned}
\end{aligned}
$$

And let $F_{k}^{(-1)}$ be defined such that 


$$
\begin{aligned}
F_{k}(z) * F_{k}^{(-1)} & =\frac{z}{1-z} \\
& =z+\sum_{n=2}^{\infty} z^{n}
\end{aligned}
$$

Then

$$
\begin{aligned}
\mathrm{J}_{\lambda, \delta}^{k} f(z) & =F_{k}^{(-1)} * f(z) \\
& =[\underbrace{\phi(z i m e s}_{k(z) * \ldots * \phi(z)} * \frac{z}{(1-z)^{\delta+1}}]^{(-1)} * f(z) \\
& =z+\sum_{n=2}^{\infty} \frac{a_{n}}{[1+(n-1) \lambda]^{k} C(\delta, n)} z^{n}, k \in \mathrm{N}_{0}, \lambda \geq 0, \delta \geq 0 \quad z \in U .
\end{aligned}
$$

Remark 1.2. When $\lambda=1, \delta=0$ we get the integral operator [3], also $k=0$ gives Noor integral operator $[7,8]$.

Some of relations for this integral operator are discussed in the next lemma.

Lemma 1.1. Let $f \in \mathrm{A}$. Then

$$
\begin{aligned}
& \text { (i) } \mathrm{J}_{\lambda, 0}^{0} f(z)=f(z), \\
& \text { (ii) } \mathrm{J}_{1,0}^{1} f(z)=\int_{0}^{z} \frac{f(t)}{t} d t .
\end{aligned}
$$

\section{Proof.}

$$
\text { (i) } \begin{aligned}
\mathrm{J}_{\lambda, 0}^{0} f(z)=z & +\sum_{n=2}^{\infty} a_{n} z^{n}=f(z), \\
\text { (ii) } \int_{0}^{z} \frac{f(t)}{t} d t & =\int_{0}^{z}\left[1+\sum_{n=2}^{\infty} a_{n} t^{n-1}\right] d t \\
& =z+\sum_{n=2}^{\infty} \frac{a_{n}}{n} z^{n} \\
& =J_{1,0}^{1} f(z) .
\end{aligned}
$$


In the following definitions, we introduce new classes of analytic functions containing the integral operator (3):

Definition 1.1. Let $f(z) \in \mathrm{A}$. Then $f(z) \in \mathrm{S}_{\lambda, \delta}^{k}(\mu)$ if and only if

$$
\mathfrak{R}\left\{\frac{z\left[\mathrm{~J}_{\lambda, \delta}^{k} f(z)\right]^{\prime}}{\mathrm{J}_{\lambda, \delta}^{k} f(z)}\right\}>\mu, 0 \leq \mu<1, z \in U .
$$

Definition 1.2. Let $f(z) \in \mathrm{A}$. Then $f(z) \in \mathrm{C}_{\lambda, \delta}^{k}(\mu)$ if and only if

$$
\mathfrak{R}\left\{\frac{\left[z\left(\mathrm{~J}_{\lambda, \delta}^{k} f(z)\right)^{\prime}\right]^{\prime}}{\left(\mathrm{J}_{\lambda, \delta}^{k} f(z)\right)^{\prime}}\right\}>\mu, 0 \leq \mu<1, z \in U
$$

Let $F$ and $G$ be analytic functions in the unit disk $U$. The function $F$ is subordinate to $G$, written $F \prec G$, if $G$ is univalent, $F(0)=G(0)$ and $F(U) \subset G(U)$. In general, given two functions $F(z)$ and $G(z)$, which are analytic in $U$, the function $F(z)$ is said to be subordination to $G(z)$ in $U$ if there exists a function $h(z)$, analytic in $U$ with

$$
h(0)=0 \text { and }|h(z)|<1 \text { for all } z \in U
$$

such that

$$
F(z)=G(h(z)) \text { for all } z \in U .
$$

Let $\phi: \mathrm{C}^{2} \rightarrow \mathrm{C}$ and let $h$ be univalent in $U$. If $p$ is analytic in $U$ and satisfies the differential subordination $\left.\phi(p(z)), z p^{\prime}(z)\right) \prec h(z)$ then $p$ is called a solution of the differential subordination. The univalent function $q$ is called a dominant of the solutions of the differential subordination, if $p \prec q$. If $p$ and $\left.\phi(p(z)), z p^{\prime}(z)\right)$ are univalent in $U$ and satisfy the differential superordination $\left.h(z) \prec \phi(p(z)), z p^{\prime}(z)\right)$ then $p$ is called a solution of the differential superordination. An analytic function $q$ is called subordinant of the solution of the differential superordination if $q \prec p$. Let $\Phi$ be an analytic function in a domain containing $f(U), \Phi(0)=0$ and $\Phi^{\prime}(0)>0$.

The function $f \in \mathrm{A}$ is called $\Phi-$ like if 


$$
\mathfrak{R}\left\{\frac{z f^{\prime}(z)}{\Phi(f(z))}\right\}>0, z \in U
$$

This concept was introduced by Brickman [9] and established that a function $f \in \mathrm{A}$ is univalent if and only if $f$ is $\Phi-$ like for some $\Phi$.

Definition 1.3. Let $\Phi$ be analytic function in a domain containing $f(U), \Phi(0)=0, \Phi^{\prime}(0)=1$ and $\Phi(\omega) \neq 0$ for $\omega \in f(U)-0$. Let $q(z)$ be a fixed analytic function in $U, q(0)=1$. The function $f \in \mathrm{A}$ is called $\Phi-$ like with respect to $q$ if

$$
\frac{z f^{\prime}(z)}{\Phi(f(z))} \prec q(z), z \in U
$$

The paper is organized as follows: Section 2 discuses the characterization properties for functions belonging to the classes $S_{k}(\mu), C_{k}(\mu)$ and Section 3, gives the subordination and superordination results involving the integral operator $\mathrm{J}_{\lambda, \delta}^{k} f(z)$. For this purpose we need to the following lemmas in the sequel.

Definition 1.4. [10] Denote by $Q$ the set of all functions $f(z)$ that are analytic and injective on $\bar{U}-E(f)$ where $E(f):=\left\{\zeta \in \partial U: \lim _{z \rightarrow \zeta} f(z)=\infty\right\}$ and are such that $f^{\prime}(\zeta) \neq 0$ for $\zeta \in \partial U-E(f)$.

Lemma 1.2. [11] Let $q(z)$ be univalent in the unit disk $U$ and $\theta$ and $\phi$ be analytic in a domain $D$ containing $q(U)$ with $\phi(w) \neq 0$ when $w \in q(U)$. Set $Q(z):=z q^{\prime}(z) \phi(q(z)), h(z):=\theta(q(z))+Q(z)$. Suppose that

1. $Q(z)$ is starlike univalent in $U$, and

2. $\mathfrak{R} \frac{z h^{\prime}(z)}{Q(z)}>0$ for $z \in U$.

If

$$
\theta(p(z))+z p^{\prime}(z) \phi(p(z)) \prec \theta(q(z))+z q^{\prime}(z) \phi(q(z))
$$


then

$$
p(z) \prec q(z)
$$

and $q(z)$ is the best dominant.

Lemma 1.3. [12] Let $q(z)$ be convex univalent in the unit disk $U$ and $\vartheta$ and $\varphi$ be analytic in a domain $D$ containing $q(U)$. Suppose that

1. $z q^{\prime}(z) \varphi(q(z))$ is starlike univalent in $U$, and

2. $\mathfrak{R}\left\{\frac{\vartheta^{\prime}(q(z))}{\varphi(q(z))}\right\}>0$ for $z \in U$.

If $\quad p(z) \in \mathrm{H}[q(0), 1] \cap Q$, with $p(U) \subseteq D$ and $\vartheta(p(z))+z p^{\prime}(z) \varphi(z)$ is univalent in $U$ and

$$
\vartheta(q(z))+z q^{\prime}(z) \varphi(q(z)) \prec \vartheta(p(z))+z p^{\prime}(z) \varphi(p(z))
$$

then $q(z) \prec p(z)$ and $q(z)$ is the best subordinant.

\section{General Properties of $\mathbf{J}_{\lambda, \delta}^{k}$}

In this section we study the characterization properties for the function $f(z) \in \mathrm{A}$ to belong to the classes $\mathrm{S}_{\lambda, \delta}^{k}(\mu)$ and $\mathrm{C}_{\lambda, \delta}^{k}(\mu)$ by obtaining the coefficient bounds.

Theorem 2.1. Let $f(z) \in \mathrm{A}$. If

$$
\sum_{n=2}^{\infty} \frac{(n-\mu)\left|a_{n}\right|}{[1+(n-1) \lambda]^{k} C(\delta, n)} \leq 1-\mu, 0 \leq \mu<1,
$$

then $f(z) \in \mathrm{S}_{\lambda}^{k},(\mu)$. The result (4) is sharp.

Proof. Suppose that (4) holds. Since 


$$
\begin{aligned}
1-\mu & \geq \sum_{n=2}^{\infty} \frac{\left|a_{n}\right||\mu-n|}{[1+(n-1) \lambda]^{k}} \\
& \geq \mu \sum_{n=2}^{\infty} \frac{\left|a_{n}\right|}{[1+(n-1) \lambda]^{k}}-\sum_{n=2}^{\infty} \frac{n\left|a_{n}\right|}{[1+(n-1) \lambda]^{k}}
\end{aligned}
$$

then this implies that

$$
\frac{1+\sum_{n=2}^{\infty} \frac{n\left|a_{n}\right|}{11+(n-1) \lambda]^{k}}}{1+\sum_{n=2}^{\infty} \frac{\left|a_{n}\right|}{[1+(n-1) \lambda]^{k}}}>\mu
$$

hence

$$
\mathfrak{R}\left\{\frac{z\left[\mathrm{~J}_{\lambda, \delta}^{k} f(z)\right]^{\prime}}{\mathrm{J}_{\lambda, \delta}^{k} f(z)}\right\}>\mu .
$$

We also note that the assertion (4) is sharp and the extremal function is given by

$$
f(z)=z+\sum_{n=2}^{\infty} \frac{[1+(n-1) \lambda]^{k} C(\delta, n)(1-\mu)}{(n-\mu)} z^{n} .
$$

Corollary 2.1. Let the assumption of Theorem 2.1. Then

$$
\left|a_{n}\right| \leq \frac{[1+(n-1) \lambda]^{k} C(\delta, n)(1-\mu)}{(n-\mu)}, \forall n \geq 2 .
$$

Corollary 2.2. Let the assumption of Theorem 2.1. Then for $\mu=\delta=0$ and $\lambda=1$

$$
\left|a_{n}\right| \leq n^{k-1}, \forall n \geq 2, k \in \mathrm{N}_{0} .
$$

In the same way we can verify the following results:

Theorem 2.2. Let $f(z) \in \mathrm{A}$. If 


$$
\sum_{n=2}^{\infty} \frac{n\left|a_{n} \| \mu+1-n\right|}{C(\delta, n)[1+(n-1) \lambda]^{k}} \leq 1-\mu, 0 \leq \mu<1,
$$

then $f(z) \in \mathrm{C}_{\lambda, \delta}^{k}(\mu)$. The result (5) is sharp.

Corollary 2.3. Let the assumption of Theorem 2.2. Then

$$
\left|a_{n}\right| \leq \frac{[1+(n-1) \lambda]^{k} C(\delta, n)(1-\mu)}{n|\mu-n+1|}, \forall n \geq 2 .
$$

Also we have the following inclusion results

Theorem 2.3. Let $0 \leq \mu_{1} \leq \mu_{2}<1$. Then $\mathrm{S}_{\lambda, \delta}^{k}\left(\mu_{1}\right) \supseteq \mathrm{S}_{\lambda, \delta}^{k}\left(\mu_{2}\right)$.

Proof. By Theorem 2.1.

Theorem 2.4. Let $0 \leq \mu_{1} \leq \mu_{2}<1$. Then $\mathrm{C}_{\lambda, \delta}^{k}\left(\mu_{1}\right) \supseteq \mathrm{C}_{\lambda, \delta}^{k}\left(\mu_{2}\right)$.

Proof. By Theorem 2.2.

Theorem 2.5. Let $0 \leq \lambda_{1} \leq \lambda_{2}$. Then $\mathrm{S}_{\lambda_{1}, \delta}^{k}(\mu) \supseteq \mathrm{S}_{\lambda_{2}, \delta}^{k}(\mu)$.

Proof. By Theorem 2.1.

Theorem 2.6. Let $0 \leq \lambda_{1} \leq \lambda_{2}$. Then $\mathrm{C}_{\lambda_{1} \delta}^{k}(\mu) \supseteq \mathrm{C}_{\lambda_{2}, \delta}^{k}(\mu)$.

Proof. By Theorem 2.2.

Moreover, we introduce the following distortion theorems.

Theorem 2.7. Let $f \in \mathrm{A}$ and satisfies (4). Then for $z \in U$ and $0 \leq \mu<1$

$$
\left|\mathrm{J}_{\lambda, \delta}^{k} f(z)\right| \geq|z|-\frac{(1-\mu)}{(2-\mu)}|z|^{2}
$$

and 


$$
\left|\mathrm{J}_{\lambda, \delta}^{k} f(z)\right| \leq|z|+\frac{(1-\mu)}{(2-\mu)}|z|^{2}
$$

Proof. By using Theorem 2.1, one can verify that

$$
(2-\mu) \sum_{n=2}^{\infty} \frac{\left|a_{n}\right|}{[1+(n-1) \lambda]^{k} C(\delta, n)} \leq \sum_{n=2}^{\infty} \frac{(n-\mu)\left|a_{n}\right|}{[1+(n-1) \lambda]^{k} C(\delta, n)} \leq 1-\mu
$$

then

$$
\sum_{n=2}^{\infty} \frac{\left|a_{n}\right|}{[1+(n-1) \lambda]^{k} C(\delta, n)} \leq \frac{1-\mu}{2-\mu}
$$

Thus we obtain

$$
\begin{aligned}
\left|\mathrm{J}_{\lambda, \delta}^{k} f(z)\right| & =\left|z+\sum_{n=2}^{\infty} \frac{a_{n}}{[1+(n-1) \lambda]^{k}} z^{n}\right| \\
& \leq|z|+\sum_{n=2}^{\infty} \frac{\left|a_{n}\right|}{[1+(n-1) \lambda]^{k}}|z|^{2} \\
& \leq|z|+\left[\frac{1-\mu}{2-\mu}\right]|z|^{2}
\end{aligned}
$$

The other assertion can be proved as follows

$$
\begin{aligned}
\left|\mathrm{J}_{\lambda, \delta}^{k} f(z)\right| & =\left|z+\sum_{n=2}^{\infty} \frac{a_{n}}{[1+(n-1) \lambda]^{k} C(\delta, n)} z^{n}\right| \\
& \geq\left|z-\sum_{n=2}^{\infty} \frac{a_{n}}{[1+(n-1) \lambda]^{k} C(\delta, n)} z^{n}\right| \\
& \geq|z|-\sum_{n=2}^{\infty} \frac{\left|a_{n}\right|}{[1+(n-1) \lambda]^{k} C(\delta, n)}|z|^{2} \\
& \geq|z|-\left[\frac{1-\mu}{2-\mu}\right]|z|^{2} .
\end{aligned}
$$

This complete the proof.

In the same way we can get the following results. 
Theorem 2.8. Let $f(z) \in \mathrm{A}$ and satisfies (5). Then for $z \in U$ and $0 \leq \mu<1$

$$
\left|\mathrm{J}_{\lambda, \delta}^{k} f(z)\right| \geq|z|-\frac{(1-\mu)}{2(2-\mu)}|z|^{2}
$$

and

$$
\left|\mathrm{J}_{\lambda, \delta}^{k} f(z)\right| \leq|z|+\frac{(1-\mu)}{2(2-\mu)}|z|^{2}
$$

Also, we have the following distortion results

Theorem 2.9. Let $f(z) \in \mathrm{A}$ and and satisfies (4). Then for $m \geq[1+(n-1) \lambda]^{k} C(\delta, n), z \in U$ and $0 \leq \mu<1$

$$
|f(z)| \geq|z|-\frac{m(1-\mu)}{(2-\mu)}|z|^{2}
$$

and

$$
|f(z)| \leq|z|+\frac{m(1-\mu)}{(2-\mu)}|z|^{2}
$$

Proof. By using Theorem 2.1, one can show that

$$
(2-\mu) \sum_{n=2}^{\infty}\left|a_{n}\right| \leq \sum_{n=2}^{\infty}(n-\mu)\left|a_{n}\right| \leq m \sum_{n=2}^{\infty} \frac{(n-\mu)\left|a_{n}\right|}{[1+(n-1) \lambda]^{k} C(\delta, n)} \leq m(1-\mu)
$$

then

$$
\sum_{n=2}^{\infty}\left|a_{n}\right| \leq \frac{m(1-\mu)}{2-\mu}
$$

Thus we obtain 


$$
\begin{aligned}
|f(z)| & =\left|z+\sum_{n=2}^{\infty} a_{n} z^{n}\right| \\
& \leq|z|+\sum_{n=2}^{\infty}\left|a_{n} \| z\right|^{2} \\
& \leq|z|+\frac{m(1-\mu)}{2-\mu}|z|^{2}
\end{aligned}
$$

The other assertion can be proved as follows

$$
\begin{aligned}
|f(z)| & \geq\left|z-\sum_{n=2}^{\infty} a_{n} z^{n}\right| \\
& \geq|z|-\sum_{n=2}^{\infty}\left|a_{n} \| z\right|^{2} \\
& \geq|z|-\frac{m(1-\mu)}{2-\mu}|z|^{2} .
\end{aligned}
$$

This completes the proof.

In the same way we can get the following results.

Theorem 2.10. Let $f(z) \in \mathrm{A}$ and and satisfies (5). Then for $z \in U$ and $0 \leq \mu<1$

$$
|f(z)| \geq|z|-\frac{m(1-\mu)}{2(2-\mu)}|z|^{2}
$$

and

$$
|f(z)| \leq|z|+\frac{m(1-\mu)}{2(2-\mu)}|z|^{2}
$$

\section{Sandwich Result.}

By making use of lemmas 1.2 and 1.3, we prove the following subordination and superordination results involving the integral operator (3). 
Theorem 3.1. Let $q \neq 0$ be univalent in $U$ such that $\frac{z q^{\prime}(z)}{q(z)}$ is starlike univalent in $U$ and

$$
\mathfrak{R}\left\{1+\left(\frac{\alpha}{\gamma}+z\right) \frac{q^{\prime \prime}(z)}{q^{\prime}(z)}-\left(\frac{\alpha}{\gamma}+z\right) \frac{q^{\prime}(z)}{q(z)}\right\}>0, \alpha, \gamma \in \mathrm{C}, \gamma \neq 0
$$

If $f \in \mathrm{A}$ satisfies the subordination

$$
(\alpha+\gamma z)\left\{\frac{1}{z}+\frac{\left[\mathrm{J}_{\lambda, \delta}^{k} f(z)\right]^{\prime \prime}}{\left[\mathrm{J}_{\lambda, \delta}^{k} f(z)\right]^{\prime}}-\frac{\Phi^{\prime}\left[\mathrm{J}_{\lambda, \delta}^{k} f(z)\right]}{\Phi\left[\mathrm{J}_{\lambda, \delta}^{k} f(z)\right]}\right\} \prec(\alpha+\gamma z) \frac{q^{\prime}(z)}{q(z)},
$$

then

$$
\frac{z\left[\mathrm{~J}_{\lambda, \delta}^{k} f(z)\right]^{\prime}}{\Phi\left[\mathrm{J}_{\lambda, \delta}^{k} f(z)\right]} \prec q(z)
$$

and $q$ is the best dominant.

Proof. Our aim is to apply Lemma 1.2. Setting

$$
p(z):=\frac{z\left[\mathrm{~J}_{\lambda, \delta}^{k} f(z)\right]^{\prime}}{\Phi\left[\mathrm{J}_{\lambda, \delta}^{k} f(z)\right]} .
$$

By computation shows that

$$
\frac{z p^{\prime}(z)}{p(z)}=1+\frac{z\left[J_{\lambda}^{k} f(z)\right]^{\prime \prime}}{\left[\mathrm{J}_{\lambda, \delta}^{k} f(z)\right]^{\prime}}-\frac{z \Phi^{\prime}\left[\mathrm{J}_{\lambda, \delta}^{k} f(z)\right]}{\Phi\left[\mathrm{J}_{\lambda, \delta}^{k} f(z)\right]}
$$

which yields the following subordination

$$
(\alpha+\gamma z) \frac{p^{\prime}(z)}{p(z)} \prec(\alpha+\gamma z) \frac{q^{\prime}(z)}{q(z)}, \alpha, \gamma \in \mathrm{C} .
$$

By setting

$$
\theta(\omega):=\frac{\alpha \omega^{\prime}}{\omega} \text { and } \phi(\omega):=\frac{\gamma}{\omega}, \gamma \neq 0,
$$


it can be easily observed that $\theta(\omega), \phi(\omega)$ are analytic in $\mathrm{C} \backslash\{0\}$ and that $\phi(\omega) \neq 0$ when $\omega \in \mathrm{C} \backslash\{0\}$. Also, by letting

$$
Q(z)=z q^{\prime}(z) \phi(q(z))=\gamma z \frac{q^{\prime}(z)}{q(z)}
$$

and

$$
h(z)=\theta(q(z))+Q(z)=\frac{\alpha q^{\prime}(z)}{q(z)}+\gamma z \frac{q^{\prime}(z)}{q(z)}=(\alpha+\gamma z) \frac{q^{\prime}(z)}{q(z)},
$$

we find that $Q(z)$ is starlike univalent in $U$ and that

$$
\mathfrak{R}\left\{\frac{z h^{\prime}(z)}{Q(z)}\right\}=\mathfrak{R}\left\{1+\left(\frac{\alpha}{\gamma}+z\right) \frac{q^{\prime \prime}(z)}{q^{\prime}(z)}-\left(\frac{\alpha}{\gamma}+z\right) \frac{q^{\prime}(z)}{q(z)}\right\}>0, \alpha, \gamma \in \mathrm{C}, \gamma \neq 0 .
$$

Then the relation (7) follows by an application of Lemma 1.2.

Corollary 3.1. Let the assumptions of Theorem 3.1 hold. Then the subordination

$$
1+\frac{z\left[\mathrm{~J}_{\lambda, \delta}^{k} f(z)\right]^{\prime \prime}}{\left[\mathrm{J}_{\lambda, \delta}^{k} f(z)\right]^{\prime}}-\frac{z\left[\mathrm{~J}_{\lambda, \delta}^{k} f(z)\right]^{\prime}}{\left[\mathrm{J}_{\lambda, \delta}^{k} f(z)\right]} \prec \frac{z q^{\prime}(z)}{q(z)},
$$

implies

$$
\frac{z\left[\mathrm{~J}_{\lambda, \delta}^{k} f(z)\right]^{\prime}}{\left[\mathrm{J}_{\lambda, \delta}^{k} f(z)\right]} \prec q(z)
$$

and $q$ is the best dominant.

Proof. By letting $\alpha=0, \gamma=1, \Phi(\omega):=\omega$.

Corollary 3.2. If $f \in \mathrm{A}$ and assume that (7) holds then 


$$
1+\frac{z\left[\mathrm{~J}_{\lambda, \delta}^{k} f(z)\right]^{\prime \prime}}{\left[\mathrm{J}_{\lambda, \delta}^{k} f(z)\right]^{\prime}}-\frac{z\left[\mathrm{~J}_{\lambda, \delta}^{k} f(z)\right]^{\prime}}{\left[\mathrm{J}_{\lambda, \delta}^{k} f(z)\right]} \prec \frac{(A-B) z}{(1+A z)(1+B z)}
$$

implies

$$
\frac{z\left[\mathrm{~J}_{\lambda, \delta}^{k} f(z)\right]^{\prime}}{\left[\mathrm{J}_{\lambda, \delta}^{k} f(z)\right]} \prec \frac{1+A z}{1+B z},-1 \leq B<A \leq 1
$$

and $\frac{1+A z}{1+B z}$ is the best dominant.

Proof. By setting $\Phi(\omega):=\omega, \gamma=1, \alpha=0 \quad$ and $\quad q(z):=\frac{1+A z}{1+B z}$ where $-1 \leq B<A \leq 1$.

Corollary 3.3. If $f \in \mathrm{A}$ and assume that (7) holds then

$$
1+\frac{z\left[\mathrm{~J}_{\lambda, \delta}^{k} f(z)\right]^{\prime \prime}}{\left[\mathrm{J}_{\lambda, \delta}^{k} f(z)\right]^{\prime}}-\frac{z\left[\mathrm{~J}_{\lambda, \delta}^{k} f(z)\right]^{\prime}}{\left[\mathrm{J}_{\lambda, \delta}^{k} f(z)\right]} \prec \frac{2 z}{1-z^{2}}
$$

implies

$$
\frac{z\left[\mathrm{~J}_{\lambda, \delta}^{k} f(z)\right]^{\prime}}{\mathrm{J}_{\lambda, \delta}^{k} f(z)} \prec \frac{1+z}{1-z},
$$

and $\frac{1+z}{1-z}$ is the best dominant.

Proof. By setting $\Phi(\omega):=\omega, \alpha=0, \gamma=1$, and $q(z):=\frac{1+z}{1-z}$.

Corollary 3.4. If $f \in \mathrm{A}$ and assume that (7) holds then

$$
1+\frac{z\left[\mathrm{~J}_{\lambda, \delta}^{k} f(z)\right]^{\prime \prime}}{\left[\mathrm{J}_{\lambda, \delta}^{k} f(z)\right]^{\prime}}-\frac{z\left[\mathrm{~J}_{\lambda, \delta}^{k} f(z)\right]^{\prime}}{\left[\mathrm{J}_{\lambda, \delta}^{k} f(z)\right]} \prec A z
$$


implies

$$
\frac{z\left[\mathrm{~J}_{\lambda, \delta}^{k} f(z)\right]^{\prime}}{\mathrm{J}_{\lambda, \delta}^{k} f(z)} \prec e^{A z}
$$

and $e^{A z}$ is the best dominant.

Proof. By setting $\Phi(\omega):=\omega, \alpha=0, \gamma=1$, and $q(z):=e^{A z},|A|<\pi$.

Theorem 3.2. Let $q(z) \neq 0$ be convex univalent in the unit disk $U$. Suppose that

$$
\mathfrak{R}\left\{\frac{\alpha}{\gamma} q^{\prime \prime}(z)-\frac{\alpha}{\gamma} \frac{q^{\prime}(z)}{q(z)}\right\}>0, \alpha, \gamma \in \mathrm{C} \text { for } z \in U
$$

and $\frac{z q^{\prime}(z)}{q(z)}$ is starlike univalent in $U$. If $\frac{z\left[\mathrm{~J}_{\lambda, \delta}^{k} f(z)\right]^{\prime}}{\Phi\left[\mathrm{J}_{\lambda, \delta}^{k} f(z)\right]} \in \mathrm{H}[q(0), 1] \cap Q$ where $f \in \mathrm{A}$,

$$
(\alpha+\gamma z)\left\{\frac{1}{z}+\frac{\left[\mathrm{J}_{\lambda, \delta}^{k} f(z)\right]^{\prime \prime}}{\left[\mathrm{J}_{\lambda, \delta}^{k} f(z)\right]^{\prime}}-\frac{\Phi^{\prime}\left[\mathrm{J}_{\lambda, \delta}^{k} f(z)\right]}{\Phi\left[\mathrm{J}_{\lambda, \delta}^{k} f(z)\right]}\right\}
$$

is univalent is $U$ and the subordination

$$
(\alpha+\gamma z) \frac{q^{\prime}(z)}{q(z)} \prec(\alpha+\gamma z)\left\{\frac{1}{z}+\frac{\left[\mathrm{J}_{\lambda, \delta}^{k} f(z)\right]^{\prime \prime}}{\left[\mathrm{J}_{\lambda, \delta}^{k} f(z)\right]^{\prime}}-\frac{\Phi^{\prime}\left[\mathrm{J}_{\lambda, \delta}^{k} f(z)\right]}{\Phi\left[\mathrm{J}_{\lambda, \delta}^{k} f(z)\right]}\right\},
$$

holds, then

$$
q(z) \prec \frac{z\left[\mathrm{~J}_{\lambda, \delta}^{k} f(z)\right]^{\prime}}{\Phi\left[\mathrm{J}_{\lambda, \delta}^{k} f(z)\right]}
$$

and $q$ is the best subordinant.

Proof. Our aim is to apply Lemma 1.3. Setting 


$$
p(z):=\frac{z\left[\mathrm{~J}_{\lambda, \delta}^{k} f(z)\right]^{\prime}}{\Phi\left[\mathrm{J}_{\lambda, \delta}^{k} f(z)\right]} .
$$

By computation shows that

$$
\frac{z p^{\prime}(z)}{p(z)}=1+\frac{z\left[\mathrm{~J}_{\lambda, \delta}^{k} f(z)\right]^{\prime \prime}}{\left[\mathrm{J}_{\lambda, \delta}^{k} f(z)\right]^{\prime}}-\frac{z \Phi^{\prime}\left[\mathrm{J}_{\lambda, \delta}^{k} f(z)\right]}{\Phi\left[\mathrm{J}_{\lambda, \delta}^{k} f(z)\right]}
$$

which yields the following subordination

$$
(\alpha+\gamma z) \frac{q^{\prime}(z)}{q(z)} \prec(\alpha+\gamma z) \frac{p^{\prime}(z)}{p(z)}, \alpha, \gamma \in \mathrm{C} .
$$

By setting

$$
\vartheta(\omega):=\frac{\alpha \omega^{\prime}}{\omega} \text { and } \varphi(\omega):=\frac{\gamma}{\omega}, \gamma \neq 0,
$$

it can be easily observed that $\vartheta(\omega), \varphi(\omega)$ are analytic in $\mathrm{C} \backslash\{0\}$ and that $\varphi(\omega) \neq 0$ when $\omega \in \mathrm{C} \backslash\{0\}$. Also, we obtain

$$
\mathfrak{R}\left\{\frac{\vartheta^{\prime}(q(z))}{\varphi(q(z))}\right\}=\Re\left\{\frac{\alpha}{\gamma} q^{\prime \prime}(z)-\frac{\alpha}{\gamma} \frac{q^{\prime}(z)}{q(z)}\right\}>0 .
$$

Then (10) follows by an application of Lemma 1.3.

Combining Theorems 3.1 and 3.2 in order to get the following Sandwich theorems

Theorem 3.3 Let $q_{1}(z) \neq 0, q_{2}(z) \neq 0$ be convex univalent in the unit disk $U$ satisfy (9) and (6) respectively. Suppose that and $\frac{z q_{i}{ }^{\prime}(z)}{q_{i}(z)}, i=1,2$ is starlike univalent in $U$. If $f \in \mathrm{A}$ and

$$
\frac{z\left[\mathrm{~J}_{\lambda, \delta}^{k} f(z)\right]^{\prime}}{\Phi\left[\mathrm{J}_{\lambda, \delta}^{k} f(z)\right]} \in \mathrm{H}\left[q_{1}(0), 1\right] \cap Q
$$




$$
(\alpha+\gamma z)\left\{\frac{1}{z}+\frac{\left[\mathrm{J}_{\lambda, \delta}^{k} f(z)\right]^{\prime \prime}}{\left[\mathrm{J}_{\lambda, \delta}^{k} f(z)\right]^{\prime}}-\frac{\Phi^{\prime}\left[\mathrm{J}_{\lambda, \delta}^{k} f(z)\right]}{\Phi\left[\mathrm{J}_{\lambda, \delta}^{k} f(z)\right]}\right\}
$$

is univalent in $U$ and the subordination

$$
(\alpha+\gamma z) \frac{q_{1}{ }^{\prime}(z)}{q_{1}(z)} \prec(\alpha+\gamma z)\left\{\frac{1}{z}+\frac{\left[\mathrm{J}_{\lambda, \delta}^{k} f(z)\right]^{\prime \prime}}{\left[\mathrm{J}_{\lambda, \delta}^{k} f(z)\right]^{\prime}}-\frac{\Phi^{\prime}\left[\mathrm{J}_{\lambda, \delta}^{k} f(z)\right]}{\Phi\left[\mathrm{J}_{\lambda, \delta}^{k} f(z)\right]}\right\} \prec(\alpha+\gamma z) \frac{q_{2}{ }^{\prime}(z)}{q_{2}(z)}
$$

holds, then

$$
q_{1}(z) \prec \frac{z\left[\mathrm{~J}_{\lambda, \delta}^{k} f(z)\right]^{\prime}}{\Phi\left[\mathrm{J}_{\lambda, \delta}^{k} f(z)\right]} \prec q_{2}(z)
$$

and $q_{1}(z)$ is the best subordinant and $q_{2}(z)$ is the best dominant.

\section{Acknowledgement}

The work here was supported by UKM-ST-06-FRGS0107-2009, MOHE Malaysia. The authors also would like to thank the anonymous referee for the informative and creative comments given to the article.

\section{References}

[1] Al-Shaqsi, K. \& Darus, M., Differential subordination with generalized derivative operator. (to appear in AJMMS)

[2] Darus, M. \& Ibrahim, R., Generalization of differential operator, Journal of Mathematics and Statistics 4(3), pp. 138-144, 2008.

[3] Să lă gean, G.S., Subclasses of univalent functions, Lecture Notes in Math., 1013, Springer-Verlag, Berlin, pp. 362-372, 1983.

[4] Ruscheweyh, S., New criteria for univalent functions, Proc. Amer. Math. Soc., 49, 109-115, 1975.

[5] Al-Oboudi, F.M., On univalent functions defined by a generalized $S$ ă lă gean operator, I.J.M.M.S, 27, pp. 1429-1436, 2004.

[6] Al-Shaqsi, Darus, M., An operator defined by convolusion involving polylogarthms functions, Journal of Mathematics and Statistics, 4(1), pp. 46-50, 2008. 
[7] Noor, K.I., On new classes of integral operators, J.Nat.Geomet. 16, pp. 71-80, 1999.

[8] Noor, K.I. \& Noor, M.A., On integral operators, J. of Math. Analy. and Appl. 238, pp. 341-352, 1999.

[9] Brickman, L., $\Phi-$ like analytic functions, I.Bull. Amer. Math. Soc. 79, pp. 555-558, 1973.

[10] Bulboaca, T., Classes of first-order differential superordinations, Demonstr.Math. 35(2), pp. 287-292, 2002.

[11] Miller, S.S. \& Mocanu, P.T., Subordinants of differential superordinations, Complex Variables, 48(10), pp.815-826, 2003.

[12] Miller, S.S. \& Mocanu, P.T., Differential Subordinantions: Theory and Applications. Pure and Applied Mathematics, 225, Dekker, New York, 2000. 